\title{
Efficacy of Interventions Based on Applied Behavior Analysis for Autism Spectrum Disorder: A Meta-Analysis
}

\author{
Qian $\mathrm{Yu}^{1} \bowtie$, Enyao $\mathrm{Li}^{1}$, Liguo $\mathrm{Li}^{1}$, and Weiyi Liang ${ }^{2}$ \\ ${ }^{1}$ Department of Child Rehabilitation, The Fifth Affiliated Hospital of Zhengzhou University, Zhengzhou, China \\ 2Department of Rehabilitation, Peking University Shenzhen Hospital, Shenzhen, China
}

\begin{abstract}
Objective To systematically evaluate evidence for the use of interventions based on appied behavior analysis (ABA) to manage various symptoms of children with autism spectrum disorder (ASD).

Methods Sensitivity analyses were conducted by removing any outlying studies and subgroup analyses were performed to compare the effectiveness of ABA and early start denver model (ESDM), picture exchange communication systems (PECS) and discrete trial training (DTT). Results 14 randomized control trials of 555 participants were included in this meta-analysis. The overall standardized mean difference was $\mathrm{d}=-0.36(95 \% \mathrm{CI}-1.31,0.58 ; \mathrm{Z}=0.75, \mathrm{p}=0.45)$ for autism general symptoms, $\mathrm{d}=0.11(95 \% \mathrm{CI}-0.31,0.54 ; \mathrm{Z}=0.52, \mathrm{p}=0.60)$ for socialization, $\mathrm{d}=0.30$ (95\% CI $-0.02,0.61 ; \mathrm{Z}=1.84, \mathrm{p}=0.07$ ) for communication and $\mathrm{d}=-3.52$ (95\% CI $-6.31,-0.72 ; \mathrm{Z}=2.47$, $\mathrm{p}=0.01)$ for expressive language, $d=-0.04(95 \% \mathrm{CI}-0.44,0.36 ; \mathrm{Z}=0.20, \mathrm{p}=0.84)$ for receptive language. Those results suggested outcomes of socialization, communication and expressive language may be promising targets for ABA-based interventions involving children with ASD. However, significant effects for the outcomes of autism general symptoms, receptive language, adaptive behavior, daily living skills, IQ, verbal IQ, nenverbal IQ, restricted and repetitive behavior, motor and cognition were not observed.
\end{abstract}

Conclusion The small number of studies included in the present study limited the ability to make inferences when comparing ABA, ESDM, PECS and DTT interventions for children with ASD.

Psychiatry Investig 2020;17(5):432-443

Key Words Autism, Children, Applied behavior analysis, Meta-analysis.

\section{INTRODUCTION}

Autism spectrum disorder (ASD) is a neurodevelopmental disorder characterized by early impairments in socialization and communication, as well as restricted interests and repetitive behaviors. ${ }^{1}$ Currently, the Centers for Disease Control and Prevention (CDC) estimates that one in every 59 children has ASD. ${ }^{2}$ Although most children are diagnosed at the age of 3 years old, approximately $39 \%$ are not evaluated for the first time until after 4 years old. ${ }^{2}$

ASD is recognized as a major public health concern because of its early onset, long duration, and high levels of associated impairments. ${ }^{3}$ This impairment is attributable not only

Received: September 5, 2019 Revised: November 7, 2019

Accepted: February 22, 2020

$\triangle$ Correspondence: Qian Yu, MSc

Department of Child Rehabilitation, The Fifth Affiliated Hospital of Zhengzhou University, No.3 Kangfuqian Street, Erqi District, Zhengzhou 450000, China

Tel: +86-15517568226, E-mail: yu.q1an@yandex.com

(a) This is an Open Access article distributed under the terms of the Creative Commons Attribution Non-Commercial License (https://creativecommons.org/licenses/bync/4.0) which permits unrestricted non-commercial use, distribution, and reproduction in any medium, provided the original work is properly cited. to the core symptoms of ASD, but also to a range of co-existing conditions that individuals with ASD often experience, including emotional and behavioral problems (i.e., anxiety, compulsions, aggression destruction and uncooperative behavior), sleep problems (i.e., difficulty in falling asleep, superficial sleep, early awakening and low sleep efficiency), feeding and eating problems, gastrointestinal problems, sensory sensitivities, learning and intellectual disabilities, as well as comorbid health and mental health diagnoses. ${ }^{4}$ Compared with the core features of ASD, these co-existing conditions can be equal or greater for parents and teachers of children with ASD than the core, and have a significant impact on behavior management, learning acquisition, and the development of social relationships. ${ }^{5}$ There are many intervention approaches for treating ASD, including applied behavior analysis (ABA), diets and vitamins, floor time, holding, medication, sensory integration, speech and music therapy, special education and visual schedules. ${ }^{6,7}$ However, there is little empirical evidence to prove the effectiveness of these approaches and the available evidence shows mixed results. ${ }^{8-11}$

ABA is a scientific approach in which procedures based on 
the principles of behavior are systematically applied to identify environmental variables that influence socially significant behavior and are used to develop individualized and practical interventions. ${ }^{12,13}$ This methodology is highly effective in teaching basic communication, games, sports, social interaction, daily living and self- help skills. As the increasing number of service providers and certified professionals in the field have suggested, the ABA field has shown even more significant growth in the field of behavioral interventions for children with autism. ${ }^{1415}$ Since the mid-1980s, there has been evidence that $\mathrm{ABA}$ has contributed to the steady accumulation of intelligence, language and social functions in children with ASD. ${ }^{16-18}$

Nowadays, there are also several types of interventions which are based on ABA and share a common set of core features, such as Early Start Denver Model (ESDM), Picture Exchange Communication Systems (PECS), Discrete Trial Training (DTT) and Pivotal Response Treatment (PRT). ESDM uses the teaching strategies which involve interpersonal exchange and positive affect, shared engagement with real-life materials and activities, adult responsivity and sensitivity to child cues, and focus on verbal and nonverbal communication, based on a developmentally informed curriculum that addresses all developmental domains. ${ }^{19}$

PECS is a manualized program that guides children to use an exchange-based communication system, which has been a common intervention choice for nonverbal children with ASD in clinical and school settings. ${ }^{20}$ DTT consists of a series of direct and systematic instruction methods, which are used repeatedly until the children acquires the skills and focuses on analyzing the skills into small elements and units. ${ }^{21} \mathrm{PRT}$ is an intervention that focuses on arranging the environment to promote the use of target structures and then provides opportunities for children to use the target structures in natural game interactions. ${ }^{22}$ Even though these interventions have their own designs and performance forms, they are all consistent with the principles of $A B A$ and show effectiveness in different functions of children with ASD. ${ }^{19-22}$

The literature on ABA-based interventions for children with ASD has been constantly growing over the past decade. At present, there are quite a number of studies on psychosocial interventions based on ABA in children with ASD. Furthermore, comparable outcome measures were used in the study to make meta-analysis possible. This meta-analysis would include ABA-based interventions like ABA, ESDM, PECS, DTT, PRT and so on.

The primary purpose of this meta-analysis was to systematically review the evidence for the use of ABA-based interventions to manage dysfunction in children with ASD. In addition, we would also examine the differences among types of ABA- based interventions in the improvement of ASD symptoms.

\section{METHODS}

\section{Protocol registration and PRISMA guidelines}

The procedures for this meta-analysis have been registered in the PROSPERO International prospective register of systematic reviews (No. CRD42018118487), which published protocols from systematic reviews prior to the initiation of data extraction in an effort to reduce reporting bias. ${ }^{23,24}$ The methods used to conduct this study were in accordance with the Cochrane Handbook for Systematic Reviews. ${ }^{25}$ This study was designed in accordance with the PRISMA guidelines. ${ }^{26}$

\section{Information sources and search strategy}

Two independent researchers identified studies by searching electronic databases and manually searching for appropriate published studies and published system reviews. The following databases were searched: Pubmed, Embase, Web of Science, Cochrane Library, Wanfang and Weipu. The main keywords utilized in the article searches included the following: autism spectrum disorder, autism, autistic disorder, ASD; applied behavior analysis, ABA; discrete trial teaching, DTT; pivotal response treatment, PRT; picture exchange communication system, PECS; early start denver model, ESDM; psychotherap* and cognitive behavi* therap*. It was limited to the title, abstract or topic, depending on the availability of search options in each database. The search was limited to journals in English and Chinese. Additionally, the search was not limited by date. Thus, all databases were searched from the earliest indexed date to December 24, 2018.

\section{Eligibility criteria and study selection}

Studies were included if they met the following criteria: 1) the study should be a randomized controlled trial (RCT); 2) participants were between the ages of 0 and 18 years old; 3 ) participants were diagnosed with ASD; 4) the treatment used in experimental group was based on / derived from applied behavior analysis (DTT, PST, PECS, ESDM and so on); 5) the treatment used in control group was conventional intervention; 6) the study included at least one standardized continuous outcome measure related to autistic symptom. The final selection of studies was performed using tools provided in the Cochrane Collaboration Handbook. ${ }^{27}$

\section{Selection of outcome measures}

Outcome measures were selected depending on their validity and frequency of use. Judgement of the validity of autistic symptoms measures in the ASD population was based on two methodologically rigorous reviews which were recently pub- 
lished. This study mainly selected outcomes related to highfrequency autistic symptoms (used more than 3 times in all included researches). Therefore, the general symptomatic outcomes of ASD, including socialization outcomes, communication outcomes, expressive language outcomes, receptive language outcomes, adaptive behavior outcomes, daily living skills outcomes and intelligence quotient (IQ) outcomes, were finally selected in this study. The selected indicators of general symptom outcomes for ASD were Mullen Scales of Early Learning (MSEL), Autism Diagnostic Observation Schedule (ADOS), Assessment of Basic Language and Learning Skills (ABLLS), Aberrant Behavior Checklist (ABC), The Autism Diagnostic Interview-Revised (ADI-R), Vineland Adaptive Behavior Scales (VABS), Autism Treatment Evaluation Checklist (ATEC) and Childhood Autism Rating Scale (CARS). The selected measures for socialization outcome were ADI-R and VABS. The selected measures for communication outcome were VABS and Psychoeducational Profile (C-PEP). The selected measures for expressive language outcome were MSEL, ADOS and Reynell Developmental Language Scales (RDLS). The selected measures for receptive language outcome were RDLS and MSEL. The selected measures for adaptive behavior outcome were VABS and C-PEP. VABS was also used for daily living skills outcome measure. In addition, Differential Ability Scales (DAS) and Stanford-Binet Intelligence Scale (SBIS) were chosen as measures of IQ outcomes.

If two of the selected outcome measures were used in a study, one of them was chosen for analysis.

\section{Data collection process and risk of bias within studies}

Data extraction and risk of bias assessment were performed according to the Cochrane Collaboration Guidelines. All references found by the search strategy were gathered by the reference management program EndNote X6 (Thomson Reuters, New York City, USA). All citation sourced from the search strategy were transferred to EndNote X6. The first author conducted the systematic search and the second author verified inclusion/ exclusion of a subset of studies. The two authors independently screened the originally selected studies and agreed on which studies should be selected for the review. Data extraction and risk of bias assessment were conducted independently by the first and second authors. In the event of a disagreement, resolutions were reached in discussion with the third referees, if necessary following inspection of the full paper. The Cochrane Collaborative tool was used to assess the risk of bias in each included study. The tool included the following domains: sequence generation, allocation concealment, blinding of participants and personnel, blinding of outcome assessment, complete outcome data, selective outcome reporting, and other sources of bias. Studies were allocated to three categories according to our judgment of each area or potential risk of bias: low risk of bias, unclear risk of bias and high risk of bias. Only methodological strengths and weaknesses associated with the results of this meta-analysis were considered when assessing the risk of bias. Whether the study should be included in the meta-analysis is judged individually based on the results of the risk of bias assessments, excluding those with higher bias risk.

Selection bias was assessed based on adequate description of random sequence generation and concealment of treatment group allocation. In order to maintain the highest level of scientific and methodological rigor, it was determined that only RCTs would be included in this review. Thus, selection bias would only come from treatment allocation. Due to the nature of interventions, blinding of participants and personnel was not feasible in any of the included studies. Thus, all studies had a high risk of performance bias. Attrition bias was assessed by examining the reports of withdrawals and drop-outs. Outcome data were considered complete if there were no missing pre- or post-treatment data, or if the study authors had carried out an intent-to-treat analysis. Therefore, reporting bias was evaluated purely based on evidence of selective outcome reporting provided in the study reports. There was no exclusion study based on bias risk assessment.

\section{Summary measures and syntheses of results}

Data syntheses were performed using Review Manager version 5.3 (Cochrane Collaboration software). We assessed continuous data and analyzed continuous data based on the basis of the available means and standard deviations. There was no clear evidence that the distribution was biased. Assuming that two or more studies were found to be suitable for inclusion and that those studies were considered to be satisfactory, a meta-analysis of the results was performed. Since the studies measured several outcomes in a nonuniform manner, outcome data were synthesized using standardized mean difference (mean/standard deviation) for both intervention and control group.

Higgin's $\mathrm{I}^{2}$ test was used to describe the impact of heterogeneity on the effect estimates in percentage terms. It was chosen over Cochrane's Q Test (a value of 0.10 used as a cut-off for significance) since the latter had low power when there were few studies. Higgins et al. ${ }^{28}$ proposed a tentative classification of $\mathrm{I}^{2}$ values with the purpose of helping to interpret its magnitude. Thus, percentages of around 25\% $\left(\mathrm{I}^{2}=25\right), 50 \%$ $\left(\mathrm{I}^{2}=50\right)$, and $75 \%\left(\mathrm{I}^{2}=75\right)$ would mean low, medium, and high heterogeneity, respectively. And a random-effect model was chosen to estimate the effect of intervention. ${ }^{29}$ 


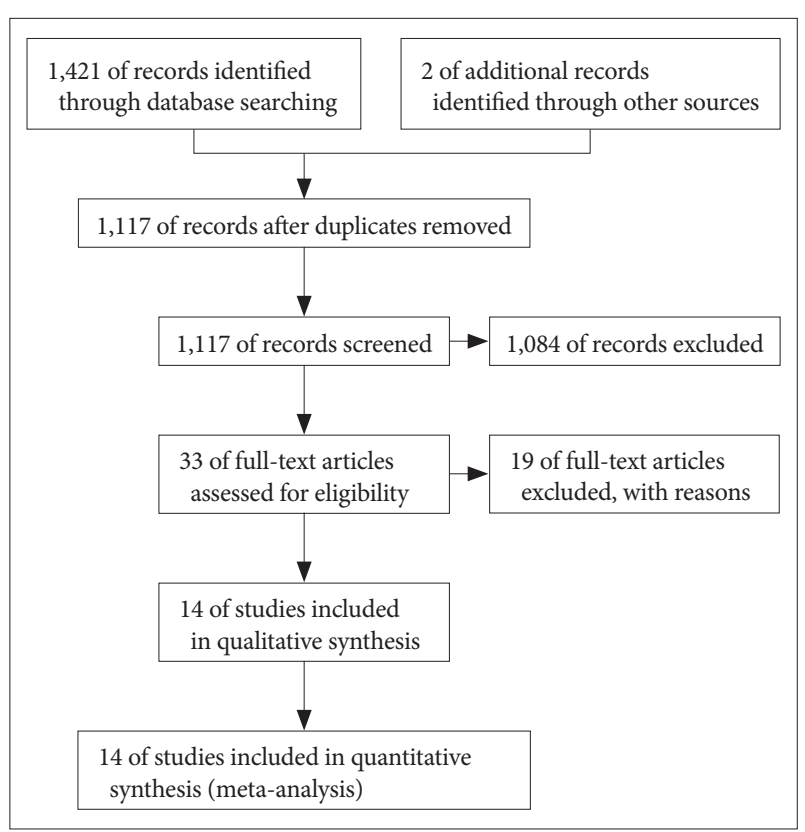

Figure 1. PRISMA flow diagram of study selection.

\section{Additional analysis}

Due to the relatively limited research addressing treatment options based on ABA for children and adolescents with ASD, it was deemed appropriate to include studies that used applied behavior analysis (ABA), discrete trial teaching (DTT), pivotal response treatment (PRT), picture exchange communication system (PECS) or early start denver model (ESDM) as intervention in experimental group. To compare the effectiveness of these delivery methods, a subgroup analysis was conducted by comparing the confidence intervals of the summary estimates in these subgroups (ABA group, DTT group, PRT group, PECS group, and ESDM group). No overlap or minimal overlap between the confidence intervals was considered statistically significant. Only subgroup analysis of the result measurements was performed if the overall summary estimates were significant.

Because of the small number of studies in each review category, it is not possible to formally assess publication bias through funnel plots or statistical tests. ${ }^{30,31}$ In order to analyze the impact of outlying studies on summary estimates, sensitivity analysis was carried out by removing each type of outlier studies.

\section{RESULTS}

\section{Study selection}

Flow diagram of the search results was shown in Figure 1. 1,421 records were identified through database searching and 2 of additional records were identified from published systematic review. After removing 306 duplicated records, 1,117 records were screened based on the title and abstract, 1,242 of which were excluded. 33 of full-text articles were assessed for eligibility and 19 of them were excluded for the following reasons: eight studies were not RCTs; one study could not provide full text after contacting the author; three studies only provided abstracts of conference articles; four studies did not meet the requirement for participants; three studies did not meet the requirement of interventions in the control group; one study did not include relevant outcomes. Finally, 14 RCTs were included in this review and meta-analysis. ${ }^{32-45}$

\section{Study characteristics}

A summary of study characteristics could be found in Table 1. A total of 555 participants (278 of experimental groups and 277 of control group) aged 6 to 102 months were included. Participants were composed of American, European, Latino, Asian, African and multiracial people. All participants in 14 studies had diagnosis of ASD by clinicians with the ADOS, the Autism Diagnostic Interview-Revised (ADI-R), the Diagnostic and Statistical Manual of Mental Disorders, Fourth Edition (DSM-VI) or the Diagnostic and Statistical Manual of Mental Disorders, Fifth Edition (DSM-5). ${ }^{32-45}$ Each study included at least one standardized continuous outcome measure related to autistic symptoms, such as socialization, communication, adaptive behavior, language, verbal IQ, non-verbal IQ, inappropriate speech, response, imitation, irritability, noncompliance, motor, body use, activity level, daily living skills, selfhelp, IQ, cognitive, early-learning, visual reception, general impression and so on. The scales with higher using frequency were ADI-R, ADOS, MSEL, VABS, ABC, CARS, ABLLS, ATEC and C-PEP.

Five studies used ABA-based intervention, ${ }^{36,38,40-42}$ one study used DTT, ${ }^{37}$ five studies used ESDM ${ }^{32-34,39,43}$ and three studies were found to use PECS. ${ }^{35,44,45}$ Eight studies were administrated by trained therapists, ${ }^{32,33,36,40-42,44,45}$ while five by teachers $^{35,37-39,43}$ and one by parents. ${ }^{34}$ Seven studies encouraged parents or caregivers to assist with generalization of acquired skills to the home environment and one of them also needed parents or caregivers to cooperate with therapists on home visit and supervision. ${ }^{32,33,36,39-41,44}$ Dawson et al. ${ }^{33}$ provided continuous training for parents during semimonthly meeting to help them use the ESDM strategy in their daily activities. ${ }^{32}$ The duration of each session was 30 to 120 minutes and the duration of the intervention was between 2 and 36 months. Intervention settings varied in different studies, such as center, elementary school, mainstream school, institution, kindergarten, department of developmental-behavioral pediatrics in hospital and home. All studies were approved by local Institutional Review Board and informed consents were obtained from the participants' parents. Gordon et al. ${ }^{35}$ assigned 


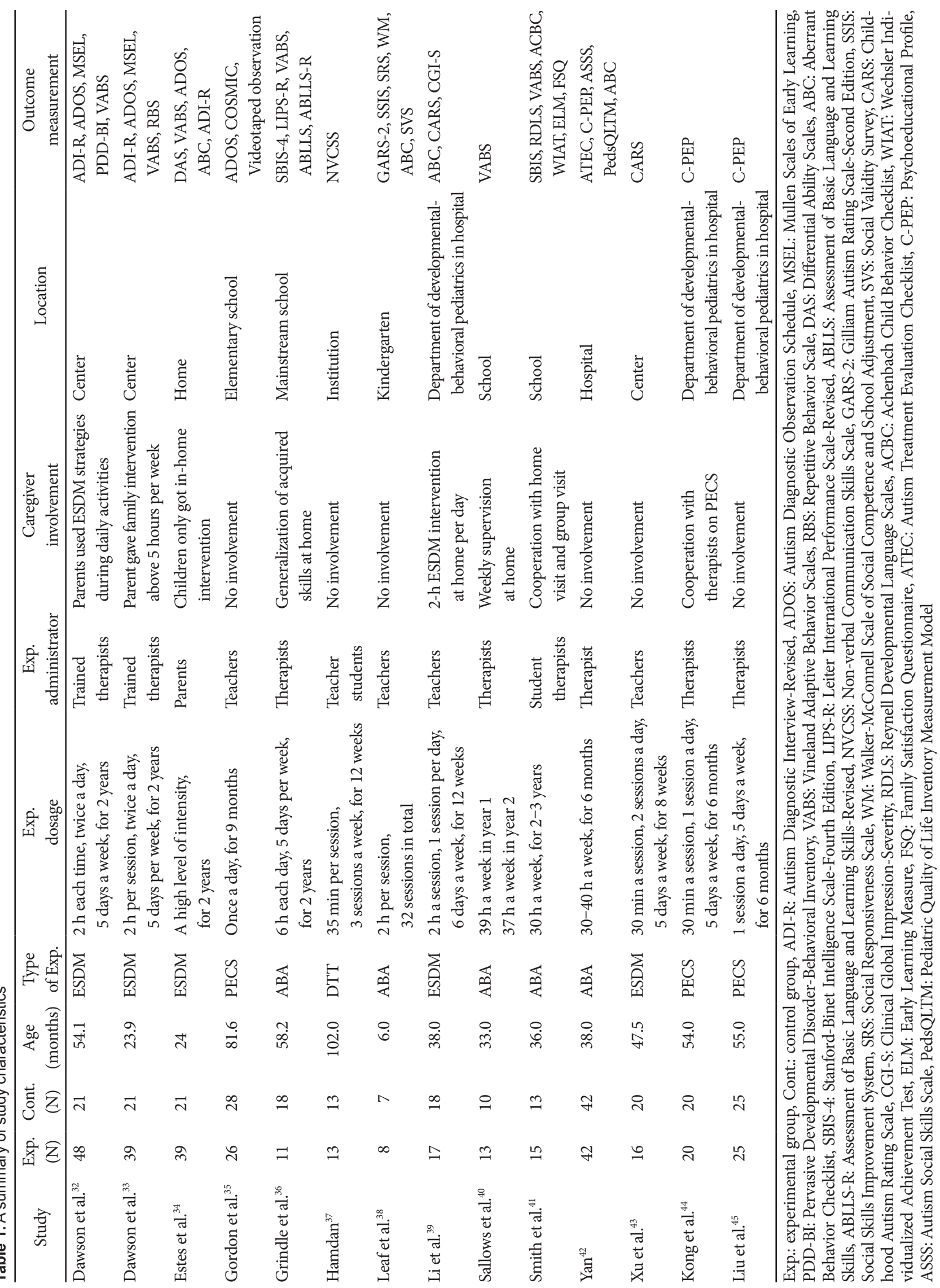


all participants into three intervention groups. The patients in immediate treatment group (ITG; five class groups, 26 children) received training immediately after baseline assessment. The patients in delayed treatment group (DTG; six class groups, 30 children) received training about 9 months later and immediately after the second assessment. The patients in no-treatment group (NTG; six class groups, 28 children) received no training. In this review, we only selected the patients in immediate treatment group and no-treatment group. A summary of study characteristics can be found in Table 1.

\section{Risk of bias within studies}

Selection bias (random sequence generation and allocation concealment)

All of the included studies were performed with adequate random sequence generation, either manually generated or computer-generated. Dawson et al. ${ }^{32}$ used random permuted blocks (Fourth Edition), while Li et al., ${ }^{39}$ Yan et al. ${ }^{42}$ and Kong et al. ${ }^{44}$ used randomized digital table. Dawson et al., ${ }^{32}$ Gordon et al., ${ }^{35}$ Hamdan et al., ${ }^{37}$ Leaf et al. ${ }^{38}$ Li et al., ${ }^{39}$ Sallows et al., ${ }^{40}$ Smith et al. ${ }^{41}$ Yan et al. ${ }^{42} \mathrm{Xu}$ et al. ${ }^{43}$ and Kong et al. ${ }^{44}$ performed adequate allocation concealment. The remainder of the included studies indicated that allocation concealment was implemented, but did not provide sufficient information about the concealment method.

Performance and detection bias (blinding of participants, personnel and outcome assessment)

As previously stated, blinding of participants and personnel was not possible in any of the included studies. And in all studies, clinicians rating scales were blind to treatment allocation so these outcome measures were considered to have a low risk of detection bias.

\section{Attrition and reporting bias (incomplete outcome data} and selective outcome reporting)

Dawson et al. ${ }^{32}$ was considered to have a high risk of attrition bias due to the deletion of missing data from the study analysis. The remainder of the included studies were deemed to have complete outcome data. There was no evidence of selective outcome reporting in any of the studies included. Risk of bias within studies is shown in Figure 2.

\section{Outcome of general symptoms of ASD}

Eleven studies reported the outcome of general symptoms of ASD and we rated the overall quality of the evidence as moderate..$^{34-36,38-45}$ These studies reported 434 participants (215 in the experimental condition and 219 in the control condition). The overall standardized mean difference (SMD)

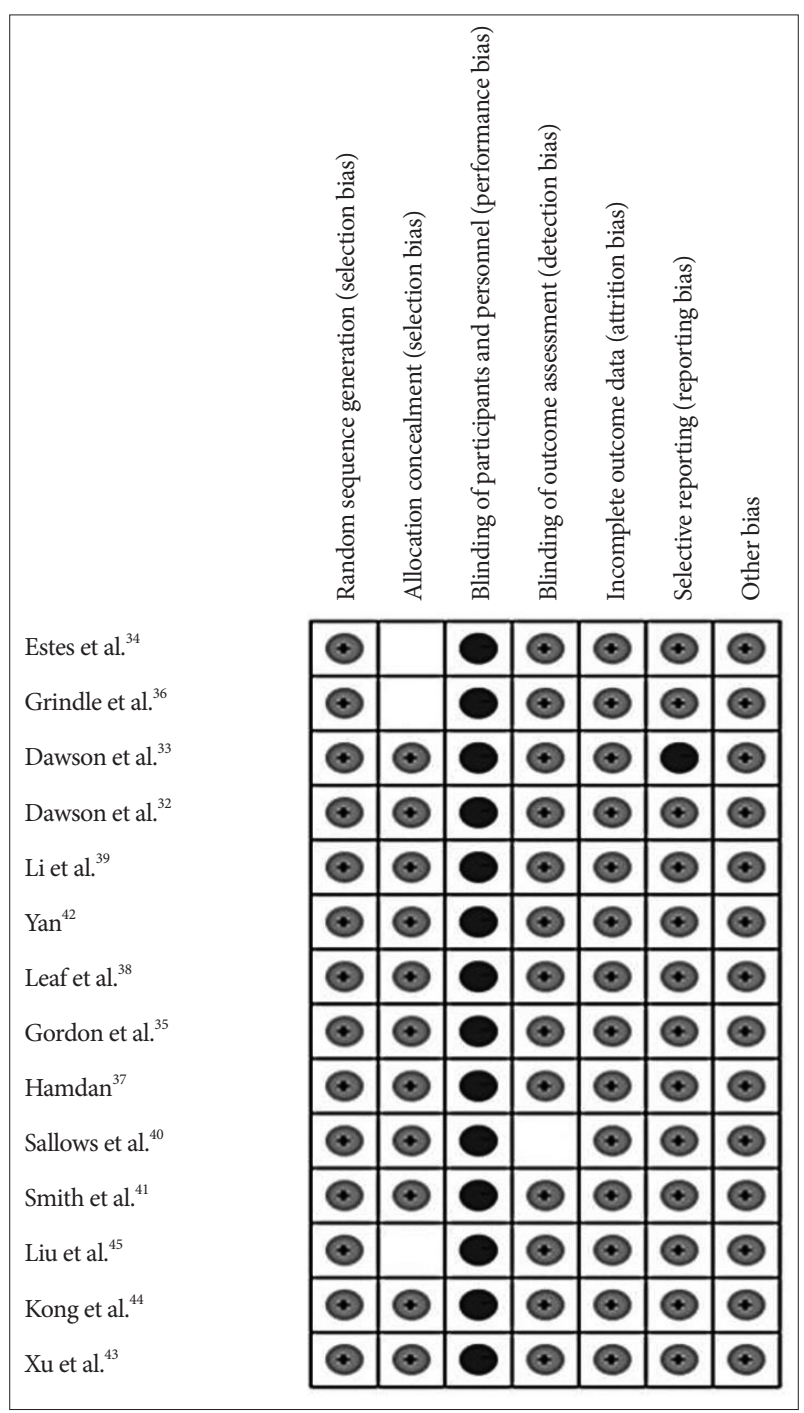

Figure 2. Risk of bias within studies.

was $\mathrm{d}=-0.36(95 \% \mathrm{CI}-1.31,0.58 ; \mathrm{Z}=0.75, \mathrm{p}=0.45)$ with no significant difference between the experimental and control conditions. There were high levels of heterogeneity across included studies $\left(\mathrm{I}^{2}=94 \%\right)$. A forest plot illustrating these results was included in Figure 3.

A subgroup analysis was carried out on ABA-based intervention $^{36,38,40-42}$ versus PECS intervention ${ }^{35,44,45}$ versus ESDM intervention ${ }^{34,39,43}$ to compared the outcome of general symptoms of ASD. There was no significant difference in the effectiveness of interventions among ABA subgroup, ESDM subgroup and ESDM subgroup. In the ABA-based intervention subgroup, the overall SMD was $\mathrm{d}=-0.12$ (95\% CI $-1.34,1.10$; $\mathrm{Z}=0.19, \mathrm{p}=0.85)$ with on significant difference between experimental and control conditions. ${ }^{36,38,40-42}$ As the high levels of heterogeneity existed, we found that the SDM reported by Sallows et $a{ }^{40}{ }^{40}$ was an outlier. Therefore, we carried out a sensitivity analysis by removing the study of Sallows et al. ${ }^{40}$ So 


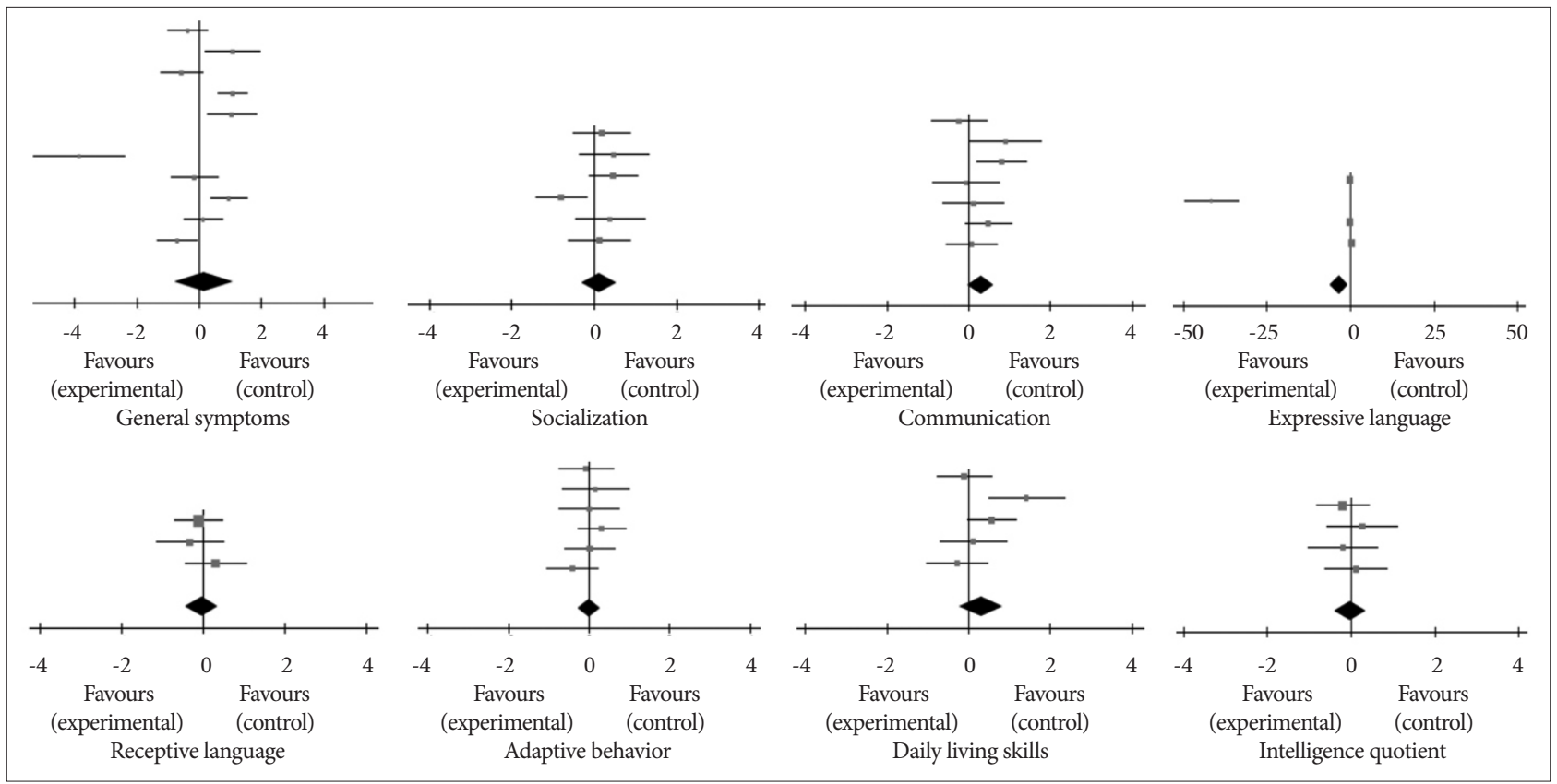

Figure 3. Forest plots.

that the overall SMD in ABA-based intervention subgroup was changed to 0.67 (95\% CI $-0.06,1.29 ; \mathrm{Z}=2.14, \mathrm{p}=0.03)$ and the difference between intervention and control conditions was significant.

In the PECS intervention subgroup, the overall SMD was $\mathrm{d}=-3.67$ (95\% CI -7.88, 0.54; $\mathrm{Z}=1.71, \mathrm{p}=0.09){ }^{35,44,45}$ There were no significant differences between experimental and control conditions and the levels of heterogeneity among studies were high $\left(I^{2}=98 \%\right)$. After conducting sensitivity analysis by removing the studies of Gordon et al., ${ }^{35}$ Liu et al. ${ }^{45}$ and Kong et al. ${ }^{44}$ respectively, the insignificance between experimental and control groups remained $(\mathrm{p}=0.16 ; \mathrm{p}=0.32 ; \mathrm{p}=0.33)$.

In the ESDM intervention subgroup, the overall SMD was $\mathrm{d}=-0.55$ (95\% CI $-0.92,-0.17 ; \mathrm{Z}=2.86, \mathrm{p}=0.04)$ with significant difference between the experimental and control conditions. ${ }^{34,39,43}$ There was no significant heterogeneity across studies $\left(\mathrm{I}^{2}=0 \%, \mathrm{p}=0.76\right)$.

\section{Outcome of socialization}

Six studies reported the outcome of socialization and we rated the overall quality of the evidence as moderate. ${ }^{32-34,36,40,41}$ These studies reported 200 participants (101 in the experimental condition and 99 in the control condition). The overall SMD was $\mathrm{d}=0.11(95 \% \mathrm{CI}-0.31,0.54 ; \mathrm{Z}=0.52, \mathrm{p}=0.60)$ and there were moderate levels of heterogeneity across studies $\left(I^{2}=55 \%, p=0.05\right)$. Since there was significant baseline imbalance [Mean (SD): Experimental group $=-0.8$ (4.7), Control group=3.4 (5.7); $\mathrm{d}=-0.80,95 \% \mathrm{CI}-1.41,-0.18$ ] in the study of Dawson et al., ${ }^{32}$ we performed sensitivity analysis by remov- ing this study. The sensitivity analysis altered the results in terms of statistical significance between experimental group and control group $(\mathrm{Z}=2.01, \mathrm{p}=0.04)$ and heterogeneity among studies $\left(\mathrm{I}^{2}=0 \%, \mathrm{p}=0.94\right)$. A forest plot illustrating these results was included in Figure 3.

Subgroup analysis was also conducted to compare ABAbased intervention ${ }^{36,40,41}$ and ESDM intervention. ${ }^{32-34}$ It was noted that there was no study used PECS or DTT intervention to report outcomes of socialization. There was no significance in the effectiveness of interventions between $A B A$ subgroup and ESDM subgroup. In the ABA-based intervention subgroup, there was no significant difference $(\mathrm{p}=0.60)$ between experimental and control groups and its heterogeneity was insignificant $\left(\mathrm{I}^{2}=0 \%, \mathrm{p}=0.81\right)$. $^{36,40,41} \mathrm{In}$ the ESDM intervention subgroup, there was still no significance $(\mathrm{p}=0.90)$ between experimental and control conditions and there were moderate levels of heterogeneity across studies $\left(\mathrm{I}^{2}=78 \%, \mathrm{p}=0.01\right) .{ }^{32-34} \mathrm{Al}-$ though the study of Dawson et al. ${ }^{32}$ was regarded as an outlier and was removed to conduct a sensitivity analysis, the insignificance between experimental and control conditions remained ( $\mathrm{p}=0.13$ ) with no significant heterogeneity among studies $\left(\mathrm{I}^{2}=0 \%, \mathrm{p}=0.54\right)$.

\section{Outcome of communication}

Seven studies reported the outcome of communication and we rated the overall quality of evidence as moderate. ${ }^{33,34,36,40,41,44,45}$ These studies reported 246 participants (122 in the experimental condition and 124 in the control condition). The overall SMD was $\mathrm{d}=0.30$ ( $95 \% \mathrm{CI}-0.02,0.61 ; \mathrm{Z}=1.84, \mathrm{p}=0.07)$ with no 
significance between experimental and control conditions. There were low levels of heterogeneity across studies $\left(\mathrm{I}^{2}=33 \%\right.$, $\mathrm{p}=0.18$ ).

Since Estes et al. ${ }^{34}$ study had significant baseline imbalance [Mean (SD): the experimental group $=5.3(20.2)$, the control group $=10$ (17.2); $\mathrm{d}=-0.24,95 \%$ CI $-0.92,0.43$ ], we performed a sensitivity analysis by removing Estes et al.'. ${ }^{34}$ study. The heterogeneity among studies decreased $\left(\mathrm{I}^{2}=16 \%\right.$, $\mathrm{p}=0.31$ ) and the difference between experimental and control groups changed to be significant.

Among the seven studies that reported outcome of communication, three used ABA-based intervention, ${ }^{36,40,41}$ two used ESDM $^{33,34}$ and two used PECS. ${ }^{44,45}$ A subgroup analysis to compare ABA versus ESDM versus PECS interventions was conducted and differences among subgroups were insignificant ( $\mathrm{p}=1.00$ ). In ABA, ESDM and PECS subgroups, the differences between experimental and control groups were all insignificant ( $\mathrm{p}=0.31, \mathrm{p}=0.16, \mathrm{p}=0.07)$. A forest plot illustrating these results was included in Figure 3.

\section{Outcome of expressive language}

Four studies reported the outcome of expressive language and we rated the overall quality of evidence as moderate. ${ }^{33,35,40,41}$ These studies reported 150 participants (78 in the experimental condition and 72 in the control condition). Among the four studies, two used ABA-based intervention, ${ }^{40,41}$ one used ESDM33 and one used PECS. ${ }^{35}$ Thus, we did not have adequate studies to carry out a subgroup analysis. Significant improvement was shown in the overall synthesis $(\mathrm{p}=0.01)$. The heterogeneity was significant $(\mathrm{p}<0.00001$, $\left.\mathrm{I}^{2}=97 \%\right)$. Since Gordon et al. $\mathrm{s}^{35}$ study had significant baseline imbalance [Mean (SD): experimental group $=0.9(0.2)$, control group $=7.5(0.1)$; $d=-41.62,95 \%$ CI $-49.79,-33.45]$, we performed sensitivity analysis by removing it. The sensitivity analysis altered the result of heterogeneity $\left(\mathrm{I}^{2}=0 \%, \mathrm{p}=0.39\right)$. A forest plot illustrating these results was included in Figure 3.

\section{Outcome of receptive language}

Three studies reported the outcome of receptive language and we rated the overall quality of evidence as moderate. ${ }^{33,40,41}$ These studies reported 96 participants (52 in the experimental condition and 44 in the control condition). Two of these studies chose ABA-based intervention ${ }^{40,41}$ and one chose ESDM intervention. ${ }^{33}$ There was not significant heterogeneity across studies $\left(\mathrm{I}^{2}=0 \%, \mathrm{p}=0.52\right)$ and we did not find significant effectiveness in the overall synthesis ( $\mathrm{p}=0.84$ ). A forest plot illustrating these results was included in Figure 3.

\section{Outcome of adaptive behavior}

Six studies reported the outcome of adaptive behavior and we rated the overall quality of evidence as moderate. ${ }^{34,40,41,43-45}$ These studies reported 210 participants (106 in the experimental condition and 104 in the control condition). Among the six studies, two used ABA-based intervention, ${ }^{40,41}$ two used ESDM ${ }^{34,43}$ and two used PECS. ${ }^{44,45}$ The overall synthesis indicated insignificance between experimental and control conditions ( $\mathrm{p}=0.93)$ with insignificant heterogeneity $\left(\mathrm{I}^{2}=0 \%\right.$, $\mathrm{p}=0.70$ ). A subgroup analysis was conducted to compare the effectiveness of ABA, ESDM and PECS and it showed that there were no significant differences between experimental and control conditions in each subgroup ( $\mathrm{p}=0.78, \mathrm{p}=0.29$, $\mathrm{p}=0.39$ ). A forest plot illustrating these results was included in Figure 3.

\section{Outcome of daily living skills}

Five studies reported the outcome of daily living skills and we rated the overall quality of evidence as moderate. . $33,34,36,40,41^{-1}$ These studies reported 77 participants (36 in the experimental condition and 41 in the control condition). Among the five studies, three used ABA-based intervention ${ }^{36,40,41}$ and two used ESDM $^{33,34}$ The overall SMD was $\mathrm{d}=0.31$ ( $95 \% \mathrm{CI}-0.22,0.84$; $\mathrm{Z}=1.14, \mathrm{p}=0.26)$ with no significant difference between the experimental and control conditions. As there were moderate levels of heterogeneity among studies and Dawson et al. s $^{33}$ study showed significant baseline imbalance [Mean (SD): experimental group=-22.6 (11.9), control group=-28.8 (9.2)], we conduct a sensitivity analysis by removing it. It was noted that the heterogeneity among studies decreased $\left(\mathrm{I}^{2}=18 \%\right)$ and the differences between experimental and control groups remained insignificant $(\mathrm{p}=0.57)$. The subgroup analysis was used to compare the effectiveness of ABA and ESDM, and there were no significant differences between experimental and control conditions in each subgroup ( $\mathrm{p}=0.43, \mathrm{p}=0.47)$. A forest plot illustrating these results was included in Figure 3.

\section{Outcome of IQ}

Four studies reported the outcome of IQ and we rated the overall quality of evidence as moderate. ${ }^{34,36,40,41}$ These studies reported 116 participants (57 in the experimental condition and 59 in the control condition). Three of these studies chose ABA-based intervention ${ }^{36,40,41}$ and one chose ESDM intervention. ${ }^{34}$ The heterogeneity test showed insignificant differences across studies $\left(\mathrm{I}^{2}=0 \%, \mathrm{p}=0.78\right)$ and no significant effectiveness were found between experimental and control conditions in the overall synthesis $(\mathrm{p}=0.87)$. A forest plot illustrating these results was included in Figure 3.

\section{Other outcomes}

In terms of verbal IQ, nonverbal IQ, restricted and repetitive behavior, and motor and cognition, there was no signifi- 
cant difference in the effectiveness of interventions between experimental and control conditions. We did not carry on the following analysis because only two studies reported in each outcome measure $(\mathrm{p}=0.56, \mathrm{p}=0.65, \mathrm{p}=0.30, \mathrm{p}=0.32$, $\mathrm{p}=0.57$ ). For the outcomes that only one study recorded, we did not conduct any test with inadequate studies.

\section{DISCUSSION}

We performed a meta-analysis of ABA-based studies (ABA, ESDM, PECS and DTT) in this study to investigate the overall effectiveness of the intervention programmers for children with ADS, and we observed no significant effects for the outcomes of general symptoms of ASD, receptive language, adaptive behavior, daily living skills, IQ, verbal IQ, nonverbal IQ, restricted and repetitive behavior, motor and cognition. However, significant effects were shown on socialization, communication and expressive language.

This study compared three types of ABA-based interventions (ABA, ESDM and PECS). Only one study reported relevant outcomes of DTT so that we could not include it in subgroup analysis. In ABA-based intervention subgroup and ESDM intervention subgroup, there were significant differences in the effectiveness between experimental and control conditions while PECS intervention not. ABA and ESDM did not have significant differences in the effectiveness on socialization and daily living skills. Additionally, all of ABA, ESDM, and PECS had no significant differences in effects on communication and adaptive behavior. As for other outcomes, there were not available studies to include in the analyses.

This study conducted a meta-analysis of ABA-based interventions (ABA, ESDM, PECS, and DTT) for children with ASD. Although several meta-analyses have assessed intervention programs related to $\mathrm{ABA}$, all of them only chose one type of ABA-based interventions that could not comprehensively reflect the effectiveness and some of them included non-randomized controlled trials so that it would introduce significant bias in the data analysis. Moreover, the quality of evidence for all outcomes were moderate, resulting in more reliable evidence than that produced by previous studies.

The small number of available studies has been limited in the ability to make inferences in comparing the four types of ABA-based interventions and investigating each type of intervention's strengths and weaknesses in terms of important outcomes. This review also neglected the influences of participant baseline levels and parent participation.

Regarding the outcome of autism general symptoms of ASD in this study, we concluded that there was not enough evidence to support the effectiveness of ABA-based interventions for treating ASD. However, the results of subgroup analysis suggested that there was possibility of effectiveness in ABA subgroup and ESDM subgroup. A previous systematic review also showed the similar result. ${ }^{46}$ In the previous review, three types of interventions were targeted: 1) behavioral interventions-based essentially on learning theory and on ABA (limited to not only early intensive behavioral intervention, but also included ABA programs derived from it; 2) social-communication focused interventions, targeting social communication impairment, as the core symptom of ASD; 3) multimodal developmental interventions targeting a comprehensive range of children's development. In the subgroup analysis, the behavioral intervention subgroup included two studies that chose ABA-based interventions and suggested that there was not enough evidence to support the treatment effectiveness of ABA-based interventions. ${ }^{47,48}$ One of the two studies was included in our meta-analysis ${ }^{41}$ and the other study was not included because its participants had no definite diagnosis of ASD. Even though both the previous study and our study have consistent conclusion, further study is still needed to accumulate evidence on the effect on general symptoms of ASD because of limited researches.

Regarding the outcomes of socialization, communication and expressive language in this study, we concluded that there was significant effectiveness of ABA-based interventions. The results on daily living skills did not show significant effectiveness of ABA-based interventions on this outcome. The results of Makrygianni et al.s ${ }^{49}$ study were consistent with our study: ABA-based interventions were moderately to very effective in improving communication skills (Effect Size: $g=0.650$ ) and expressive language skills (Effect Size: g=0.742), moderately effective in improving socialization (Effect Size: g=0.444), lowly effective in improving daily living skills (Effect Size: $\mathrm{g}=0.138$ ). The effect size (ES) in previous studies was one of the indexes of magnitude and direction of the treatment effect. ${ }^{50-52}$ Specifically, ES constituted a quantitative assessment of the magnitude and the power of a phenomenon..$^{53}$ The type of ES, used in the present study, was the standardized mean change $\left(\mathrm{ES}_{\text {change }}\right)$ which expressed the difference between preand post-treatment measures. Hedges ${ }^{54} \mathrm{~g}$ was used to calculate the standardized mean change because it constituted a conservative estimate. For the interpretation of ES, Cohen provided Rules-of-Thumb suggesting that 0.2 represented a small ES, 0.5 represented a medium ES and 0.8 represented a large ES..$^{51}$

Studies used quasi-experimental, within-subjects, and prepost design to evaluate the efficacy of ABA-based interventions on ASD. The remaining studies used a quasi-experimental between-groups pre-post design, comparing the performance of an experimental group, receiving ABA-based intervention while a control group received an eclectic or "treatment-as- 
usual" intervention. Only two studies used a random experimental between- groups pre-post design and were included in our meta-analysis. ${ }^{40,41}$ The outcomes in some studies were based on an assessment of larger number of studies and used more rigorous analyses to estimate mean effect sizes of each outcome. However, due to the selection bias of the included studies, larger sample randomized control trials are still needed. For the outcomes of IQ, verbal IQ, nonverbal IQ, restricted and repetitive behavior and motor, we did not find relevant studies to compare and analyze.

The present study also demonstrated the insignificant effectiveness of ABA-based interventions for children with ASD on receptive language, adaptive behavior and cognition, which was consistent with the previous study. ${ }^{55}$ In the previous study, thirteen studies met the inclusion criteria and six of them were randomized comparison trials with adequate methodologic quality. Meta-analysis of 4 studies concluded it when compared with standard care. ABA intervention programs did not significantly improve the cognitive outcomes of children in the experimental group who scored a SMD of 0.38 (95\% CI 0.09 to $0.84 ; \mathrm{p}=0.11$ ), for receptive language; SMD of 0.29 ( $95 \%$ CI 0.17 to $0.74 ; \mathrm{p}=0.22$ ) or adaptive behavior; SMD of 0.39 ( $95 \%$ CI 0.16 to 0.77 ; $\mathrm{p}=0.20$ ). Among the four included studies, two studies were not eligible for this review because they excluded children with ASD who had an IQ score less than $50^{56,57}$ and the others were included in this review. ${ }^{40,41}$ Thus, currently, there is inadequate evidence that ABA-based interventions have better outcomes than standard care for children with ASD on receptive language, adaptive behavior and cognition.

Additionally, we found that long-term, comprehensive ABA-based interventions were beneficial to lifelong development of children with ASD. In Virués-Ortega's study, the results suggested that long-term, comprehensive ABA-based intervention led to (positive) medium to large effects in terms of intellectual functioning, language development, acquisition of daily living skills and social functioning in children with ASD.$^{58}$ Although favorable effects were apparent across all outcomes, language-related outcomes (IQ, receptive and expressive language, communication) were superior to nonverbal IQ, social functioning and daily living skills, with effect sizes approaching 1.5 for receptive and expressive language and communication skills. Dose-dependent effect sizes were apparent by levels of total treatment hours for language and adaptation composite scores. In Roth et al.'s9 ${ }^{59}$ study, adolescents and adults with ASD were included and the results suggested that the behavioral interventions in the areas of academic skills, adaptive skills, problem behavior interventions in the areas of academic skills, adaptive skills, problem behavior, phobic avoidance, social skills, and vocational skills had medium-to-strong effect sizes. Medium-to-high confidence in findings was noted for $81 \%$ of the studies in the meta-analysis; however, three-fourths of the reviewed studies did not include treatment integrity, which may affect the ability to draw conclusion about the effectiveness of the interventions. Therefore, it is necessary for children with ASD to ensure long-term adherence to treatment, for ABA-based interventions may have slower effect.

It was also noted that parental synchrony and sensitivity played a role in helping mediators enhance the communication and social interaction of children with $\mathrm{ASD}^{60}$ and in the effectiveness of enhancing children's reciprocity of social interaction toward others not only in Aldred et al. ${ }^{61}$ and Green et al., ${ }^{62}$ but also in the other studies. ${ }^{63-67}$ However, our study did not consider the influences of parental synchrony and sensitivity, which should be improved in the further study.

This review suggested that the outcomes of socialization, communication and expressive language may be promising targets for ABA-based interventions involving children with ASD. However, significant effects for the outcomes of general symptoms of ASD, receptive language, adaptive behavior, daily living skills, IQ, verbal IQ, nonverbal IQ, restricted and repetitive behavior, motor and cognition were not observed. The small number of studies included in the present study were limited in the ability to make inferences when comparing ABA, ESDM, PECS and DTT interventions for children with ASD and investigating the strengths and weaknesses of each type of intervention in terms of important outcomes. More methodologically rigorous researches will be necessary to ascertain the precise potential of ABA-based interventions for children with ASD.

\section{Acknowledgments}

This study was supported by the Joint Construction Project of Henan Medical Science and Technology Research Plan (No.2018020223).

\section{Conflicts of Interest}

The authors have no potential conflicts of interest to disclose.

\section{Author Contributions}

Conceptualization: Qian Yu. Data curation: Enyao Li, Liguo Li, Weiyi Liang. Formal analysis: Weiyi Liang. Investigation: Weiyi Liang. Methodology: Qian Yu. Project administration: Qian Yu. Resources: Qian Yu. Software: Weiyi Liang. Supervision: Qian Yu. Validation: Qian Yu. Visualization: Qian Yu. Writing_original draft: Weiyi Liang, Qian Yu. Writing_review \& editing: Qian Yu.

\section{ORCID iDs}

Qian Yu https://orcid.org/0000-0001-8882-1994

Enyao Li https://orcid.org/0000-0001-7804-4984

Liguo Li https://orcid.org/0000-0003-2794-6587

Weiyi Liang https://orcid.org/0000-0002-8278-355X 


\section{REFERENCES}

1. American Psychiatric Association. Diagnostic and Statistical Manual of Mental Disorders (5th Edition). Washington, DC: American Psychiatric Publishing; 2013.

2. Centers for Disease Control and Prevention. Prevalence of autism spectrum disorder among children aged 8 years - Autism and developmental disabilities monitoring network, 11 sites, United States, 2014. Morbidity and Mortality Weekly Report Surveillance Summaries, April 27th, 2018.

3. Simonoff E, Pickles A, Charman T, Chandler S, Loucas T, Baird G. Psychiatric disorders in children with autism spectrum disorders: prevalence, comorbidity, and associated factors in a population-derived sample. J Am Acad Child Adolesc Psychiatry 2008;47:921-929.

4. Maskey M, Warnell F, Parr JR, Le Couteur A, McConachie H. Emotional and behavioural problems in children with autism spectrum disorder. J Autism Dev Disord 2012;43:851-859.

5. Pearson DA, Loveland KA, Lachar D, Lane DM, Reddoch SL, Mansour R, et al. A comparison of behavioral and emotional functioning in children and adolescents with autistic disorder and PDD-NOS. Child Neuropsychol 2006;12:321-333.

6. Green VA, Pituch KA, Itchon J, Choi A, O’Reilly M, Sigafoos J. Internet survey of treatments used by parents of children with autism. Res Dev Disabil 2006;27:70-84.

7. Hess KL, Morrier MJ, Heflin LJ, Ivey ML. Autism treatment survey: services received by children with autism spectrum disorders in public school classrooms. J Autism Dev Disord 2008;38:961-971.

8. Foxx RM. Applied behavior analysis treatment of autism: the state of art. Child Adolesc Psychiatr Clin N Am 2008;17:821-834.

9. Howlin P. Interventions for people with autism: recent advances. Adv Psychiatr Treat 1997;3:94-102.

10. Schechtman MA. Scientifically unsupported therapies in the treatment of young children with Autism Spectrum Disorders. Pediatr Ann 2007;36:497-505.

11. Smith T. Outcome of early intervention for children with autism. Clin Psychol Sci Pract 1999;6:33-49.

12. Baer DM, Wolf MM. Some still-current dimensions of applied behavior analysis. J Appl Behav Anal 1987;20:313-327.

13. Baer DM, Wolf MM, Risley TR. Some current dimensions of applied behavior analysis. J Appl Behav Anal 1968;1:91-97.

14. Cooper JO, Heron TE, Heward WL. Definition and Characteristics of Applied Behavior Analysis. In: Cooper JO, Heron TE, Heward WL, Editors. Applied Behavior Analysis. 2nd Edition. Upper Saddle River, NJ: Pearson, 2007, p.2-23.

15. Shook GL. An examination of the integrity and future of the Behavior Analyst Certification Board credentials. Behav Modif 2005;29:562574.

16. Fenske EC, Zalenski S, Krantz PJ, McClannahan LE. Age at intervention and treatment outcome for autistic children in a comprehensive intervention program. Res Dev Disabil 1985;549-558.

17. Lovaas OI. Behavioral treatment and normal educational and intellectual functioning in young autistic children. J Consult Clin Psychol 1987;55:3-9.

18. Remington B, Hastings RP, Kovshoff H, degli Espinosa F, Jahr E, Brown T, et al. Early intensive behavioral intervention: outcomes for children with autism and their parents after two years. Am J Ment Retard 2007;112:418-438.

19. Smith M, Rogers S, Dawson G. The Early Start Denver Model: A Comprehensive Early Intervention Approach for Toddlers with Autism. In: Handleman JS, Harris SL, Editors. Preschool Education Programs for Children with Autism. 3. Austin, TX: Pro-Ed Corporation Inc, 2008, p.65-101.

20. Angermeier K, Schlosser RW, Luiselli JK, Harrington C, Carter B. Effects of iconicity on requesting with the Picture Exchange Communication System in children with autism spectrum disorder. Res Autism
Spectrum Disord 2008;2:430-446.

21. Smith T. Discrete trial training in the treatment of autism. Focus Autism Dev Disabil 2001;16:86-92.

22. Mohammadzaheri FM, Koegel LK, Rezaee M, Rafiee SM. A randomized clinical trial comparison between Pivotal Response Treatment (PRT) and structured Applied Behavior Analysis (ABA) intervention for children with autism. J Autism Dev Disord 2014;44:2769-2777.

23. Schiavo JH. PROSPERO: an international register of systematic review protocols. Med Ref Serv Q 2019;38:171-180.

24. Moher D, Shamseer L, Clarke M, Ghersi D, Liberati A, Petticrew M, et al. Preferred reporting items for systematic review and meta-analysis protocols (PRISMAP) 2015 statement. Syst Rev 2015;4:1.

25. Higgins JP, Green S. Cochrane Handbook for Systematic Reviews of Interventions. Chichester: Wiley Online Library; 2008.

26. Liberati A, Altman DG, Tetzlaff J, Mulrow C, Gotzsche PC, Ioannidis JPA, et al. The PRISMA statement for reporting systematic reviews and meta-analyses of studies that evaluate health care interventions: explanation and elaboration. PLos Med 2009;6:e1000100.

27. Higgins JPT, Green S. Cochrane Handbook for Systematic Reviews of Interventions Version 5.1.0. Available at: http://handbook.cochrane. org/. Accessed March 20, 2011.

28. Higgins JP, Thompson SG, Deeks JJ, Altman DG. Measuring inconsistency in meta-analyses. BMJ 2003;327:557-560.

29. DerSimonian R, Laird N. Meta-analysis in clinical trials. Control Clin Trials 1986;7:177-188.

30. Cohen J. Statistical Power Analysis in the Behavioral Sciences. 2nd Edition. Hillsdale: Lawrence Erlbaum Associates, Inc; 1988.

31. Macaskill P, Walter SD, Irwig L. A comparison of methods to detect publication bias in meta-analysis. Stat Med 2001;20:641-654.

32. Dawson G, Jones EJ, Merkle K, Venema K, Lowy R, Faja S, et al. Early behavioral intervention is associated with normalized brain activity in young children with autism. J Am Acad Child Adolesc Psychiatry 2012;51:1150-1159.

33. Dawson G, Rogers S, Munson J, Smith M, Winter J, Greenson J, et al. Randomized, controlled trial of an intervention for toddlers with autism: the Early Start Denver Model. Pediatrics 2009;125:e17-e23.

34. Estes A, Munson J, Rogers SJ, Greenson J, Winter J, Dawson G. Longterm outcomes of early intervention in 6-year- old children with autism spectrum disorder. J Am Acad Child Adolesc Psychiatry 2015;54:580587.

35. Gordon K, Pasco G, McElduff F, Wade A, Howlin P, Charman T. A communication-based intervention for nonverbal children with autism: what changes? Who benefits? J Consult Clin Psychol 2011;79:447457.

36. Grindle CF, Hastings RP, Saville M, Hughes JC, Huxley K, Kovshoff H, et al. Outcomes of a behavioral education model for children with autism in a mainstream school setting. Behav Modif 2012;36:298-319.

37. Hamdan MA. Developing a proposed training program based on discrete trial training (DTT) to improve the non-verbal communication skills in children with autism spectrum disorder (ASD). Int J Spec Educ 2018;33:579-591.

38. Leaf JB, Leaf, JA, Milne C, Taubman M, Oppenheim-Leaf M, Torres N, et al. An evaluation of a behaviorally based social skills group for individuals diagnosed with autism spectrum disorder. J Autism Dev Disord 2017;47:243-259.

39. Li HH, Li CL, Gao D, Pan XY, DU L, Jia FY. Preliminary application of early start denver model in children with autism spectrum disorder. Chin J Contemp Pediatr 2018;20:793-798.

40. Sallows GO, Graupner TD. Intensive behavioral treatment for children with autism: four- year outcome and predictors. Am J Ment Retard 2005;110:417-438.

41. Smith T, Groen AD, Wynn JW. Randomized trial of intensive early intervention for children with Pervasive Developmental Disorder. Am J Ment Retard 2000;105:269-285.

42. Yan HH. The effect of behavioral analysis therapy on children with au- 
tism and its influence on social function. Chin J Convalescent Med 2018;27:580-582.

43. Xu Y, Yao J, Yang J. Application of early start denver model for early intervention on autistic children. Chin J Clin Psychol 2017;25:188-191.

44. Kong XY, Song FX, Li H, Xu HW, Wang YN, Li Y, et al. Effect of picture exchange communication system on children with autism. Chin J Rehabil Theory Pract 2014;11:1086-1088.

45. Liu XL. The application value of picture exchange communication system in the rehabilitation and nursing of children with autism. Mod J Integr Tradit Chin West Med 2016;25:3634-3636.

46. Tachibana Y, Miyazaki C, Ota E, Mori R, Hwang Y, Kobayashi E, et al. A systematic review and meta-analysis of comprehensive interventions for pre-school children with autism spectrum disorder (ASD). PLoS One 2017;12:e0186502.

47. Smith T, Groen AD, Wynn JW. Randomized trial of intensive early intervention for children with pervasive developmental disorder. Am J Ment Retard 2000;105:269-285.

48. Reitzel J, Summers J, Lorv B, Szatmari P, Zwaigenbaum L, Georgiades $S$, et al. Pilot randomized controlled trial of a Functional Behavior Skills Training program for young children with autism spectrum disorder who have significant early learning skill impairments and their families. Res Autism Spectr Disord 2013;7:1418-1432.

49. Makrygianni MK, Gena A, Galanis P, Katoudi S. The effectiveness of applied behavior analytic interventions for children with Autism Spectrum Disorder: a meta-analytic study. Res Autism Spectr Disord 2018;51: 18-31.

50. Hedges LV, Olkin I. Statistical Methods for Meta-Analysis. San Diego, CA: Academic Press; 1985.

51. Lipsey MW, Wilson DB. Practical Meta-Analysis. Los Angeles: Sage Publications; 2001.

52. O'Mara AJ, Marsh HW, Craven RG. Meta-Analytic methods in educational research: issues and their solutions. Australia: Paper presented at Australian Association for research in education annual conference; 2005.

53. Kelley K, Preacher KJ. On effect size. Psychol Methods 2002;17:137152.

54. Hedges LV. Distribution theory for Glass's estimator of effect size and related estimators. J Educ Behav Stat 1981;6:107-128.

55. Spreckley M, Boyd R. Efficacy of applied behavioral intervention in preschool children with autism for improving cognitive, language, and adaptive behavior: a systematic review and meta-analysis. J Pediatr
2009;154:338-344.

56. Eikeseth S, Smith T, Jahr E, Eldevik S. Intensive behavioral treatment at school for 4- to 7- year-old children with autism: a one-year comparison controlled study. Behav Modif 2002;26:49-68.

57. Eikeseth S, Smith T, Jahr E, Eldevik S. Outcome for children with autism who began intensive behavioral treatment between ages 4 and 7: a comparison controlled study. Behav Modif 2007;31:264-278.

58. Virués-Ortega J. Applied behavior analytic intervention for autism in early childhood: meta-analysis, meta-regression and dose-response meta-analysis of multiple outcomes. Clin Psychol Rev 2010;30:387-399.

59. Roth ME, Gillis JM, DiGennaro Reed FD. DiGennaro Reed. A metaanalysis of behavioral interventions for adolescents and adults with autism spectrum disorders. J Behav Educ 2014;23:258-286.

60. Aldred C, Green J, Emsley R, McConachie H. Brief report: Mediation of treatment effect in a communication intervention for pre-school children with autism. J Autism Dev Disord 2012;42:447-454.

61. Aldred C, Green J, Adams C. A new social communication intervention for children with autism: pilot randomised controlled treatment study suggesting effectiveness. J Child Psychol Psychiatry 2004;45:14201430.

62. Green J, Charman T, McConachie H, Aldred C, Slonims V, Howlin P, et al. Parent-mediated communication-focused treatment in children with autism (PACT): a randomised controlled trial. Lancet 2010;375: 2152-2160.

63. Casenhiser DM, Shanker SG, Stieben J. Learning through interaction in children with autism: preliminary data from asocial-communication-based intervention. Autism 2013;17:220-241.

64. Ichikawa K, Takahashi Y, Ando M, Anme T, Ishizaki T, Yamaguchi H, et al. TEACCH-based group social skills training for children with high-functioning autism: a pilot randomized controlled trial. Biopsychosoc Med 2013;7:14.

65. Kaale A, Smith L, Sponheim E. A randomized controlled trial of preschool-based joint attention intervention for children with autism. J Child Psychol Psychiatry 2012;53:97-105.

66. Kasari C, Gulsrud AC, Wong C, Kwon S, Locke J. Randomized controlled caregiver mediated joint engagement intervention for toddlers with autism. J Autism Dev Disord 2010;40:1045-1056.

67. Lawton K, Kasari C. Brief report: longitudinal improvements in the quality of joint attention in preschool children with autism. J Autism Dev Disord 2012;42:307-312. 